\title{
The Application based on Fourier Transform in Time Lens
}

\author{
Rulin Liu ${ }^{1}$, Shuqin Guo ${ }^{1,2}$ and Dacai $\mathrm{Xu}^{1}$ \\ ${ }^{1}$ Zhejiang Provincial Key Laboratory for Signal Processing, College of Information Engineering, Zhejiang University of Technology, \\ Hangzhou 310023, Zhejiang, P.R. China \\ ${ }^{2}$ Key Laboratory of Specialty Fiber Optics and Optical Access Networks, Shanghai University, 200072 Shanghai
}

\begin{abstract}
Using the similarity between the transmission of narrow band light pulses in the dispersion media and the transmission of spatial beam in paraxial diffraction, build a time lens system similar to the space lens. Space lens can achieve compression and amplification of imaging function, and it has the nature of Fourier transform in light field. Then the time lens can also realize the compression and amplification of the light pulse and its Fourier transform. Using the function of Fourier transform in the time domain of time lens system, achieved the effect of eliminating noise and removing side lobe, improved the white noise which generally exist in the communication system and the side lobe effect which will produce in the relevant processes.
\end{abstract}

\section{Introduction}

By comparing the propagation equation (Fraunhofer diffraction) of the paraxial diffraction in free space with the dispersion equation of the narrow band light pulse in the dispersion medium transmission, we found they are very similar. When the paraxial beam is transmitted its space frequency introduced the second-order phase, and narrow band light pulse also introduced second-order phase in time frequency. In addition, in spatial optical processing, space lens can be viewed as a spatial phase modulator, and it provides a secondary phase modulation for space position variables, so people provide a secondary phase modulation for time variables using the similar principle of diffraction and dispersion transport. Similar to the spatial imaging systems, people set up a application prospects such as the optical pulse compression and amplification [7], transformation of time domain, frequency domain [8], optical signal detection and process [9-10], etc..

\section{The principles of Fourier transform using time lens}

In the system of time lens, the similarity of the signal's dispersion transmission and the diffraction of light are based on the effective ignorance of the nonlinear effects [1], high-order dispersion effect and the loss of the fiber.

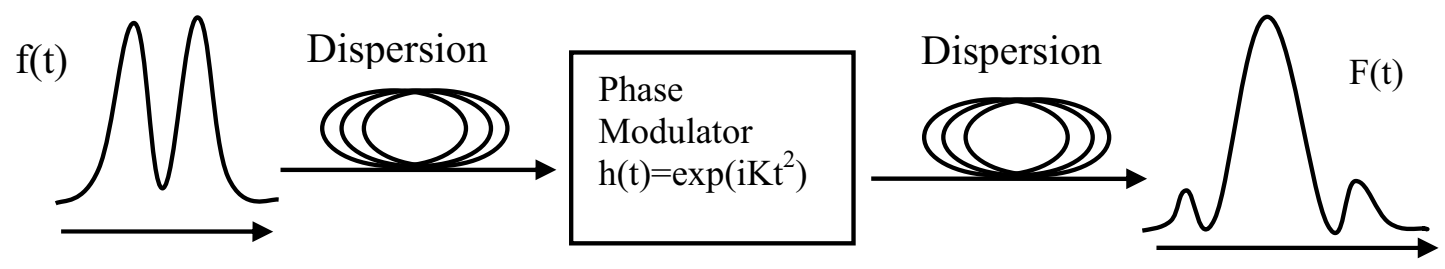

Figure 1. The structural model of time lens

time imaging system and call it the time lens [1-2].

We know the space lens can achieve the function of compression and amplification imaging and spatial Fourier transform on the focal length of the light field. Similar to the space lens, time lens can realize signal compression, amplification and Fourier transform. Then, it opens up a new path in multiple fields of optical signals. In recent years, people carried on the thorough research of time lens both at home and abroad. The theory of time lens continuously perfect [3-6], and it has broad
Figure 1 is a structural model of the time lens which is based on the spatial lens system. The system can be part of two dispersion fiber, and the phase modulator can be used the Mach-Zehnder Interferometer (MZI).

Assuming that the input signal is $\mathrm{f}(\mathrm{t})$, its spectrum is $\mathrm{F}(\mathrm{w})$, the dispersion is equal to the length of the fiber multiplied by the second-order dispersion, phase modulator $h(t)=\exp (\mathrm{iKt} 2)$. In the frequency domain, when ignoring the nonlinear effect, the loss and the high- 
order dispersion of the fiber, the transfer function of the fiber or the dispersion device is

$$
H_{D}(w)=\exp \left(\frac{i}{2} \beta_{2} L w^{2}\right)
$$

For the phase modulator $\mathrm{h}(\mathrm{t})=\exp (\mathrm{iKt} 2), \mathrm{K}$ is the phase shift coefficient of the phase modulator, its transfer function in spectrum domain is

$$
H_{\mathrm{M}}(w)=F[h(t)]=\sqrt{i \pi / K} \exp \left(\frac{-i w^{2}}{4 K}\right)
$$

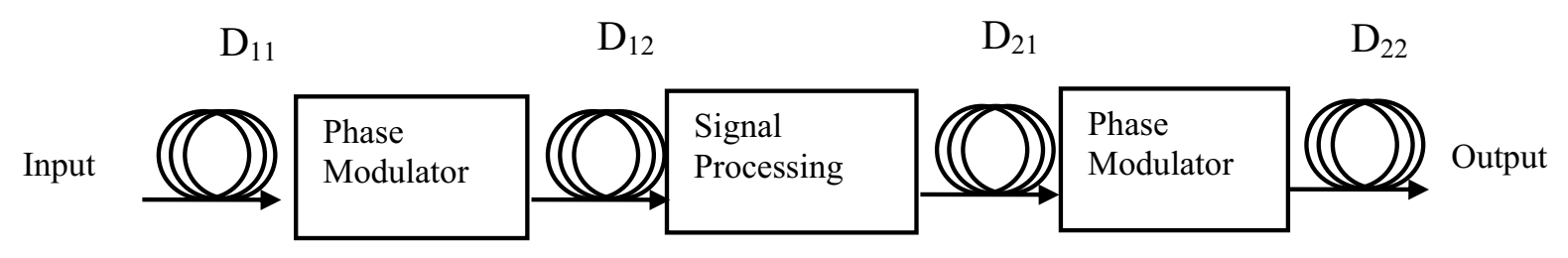

Figure 2. The structural model of double-lens

Where the $\mathrm{F}$ is the Fourier transform operator. The input signal is expressed as $s(t, 0)$, after the first fiber but before the modulator, it is expressed as $\mathrm{s}\left(\mathrm{t}, \mathrm{L}_{1}\right)$, its spectrum is $\mathrm{S}\left(\mathrm{w}, \mathrm{L}_{1}\right)$, then,

$$
\begin{aligned}
& \mathrm{S}\left(w, L_{1}\right)=\mathrm{S}(w, 0) H_{D}(w) \\
& \mathrm{s}\left(t, L_{1}\right)=F^{-1}\left[S\left(w, L_{1}\right)\right]
\end{aligned}
$$

After the modulator,

$$
\begin{gathered}
\mathrm{s}\left(t, L_{1}+M\right)=s\left(t, L_{1}\right) h(t) \\
\mathrm{S}\left(w, L_{1}+M\right)=F\left[s\left(t, L_{1}+M\right)\right]
\end{gathered}
$$

Then through the second fiber, the final signal is

$$
\mathrm{s}\left(t, L_{1}+M+L_{2}\right)=F^{-1}\left[S\left(w, L_{1}+M\right) H_{D}(w)\right]
$$

Finally, after finishing,

$$
\begin{aligned}
& \mathrm{s}\left(t, L_{1}+M+L_{2}\right)=\frac{1}{2 \pi\left|\beta_{22} L_{2}\right|} \sqrt{\frac{i \pi}{K}} S\left(\frac{t}{\left|\beta_{22} L_{2}\right|}, 0\right) * \\
& \exp \left(t^{2}\left(\frac{\beta_{21} L_{2}+\beta_{22} L_{2}}{2\left(\beta_{22} L_{2}\right)^{2}}-\frac{1}{\beta_{22} L_{2}}\right)\right)
\end{aligned}
$$

The result of formula 8 show that, when $\beta_{21} \mathrm{~L}_{1}=\beta_{22} \mathrm{~L}_{2}$, the output signal is the Fourier transform waveform of the input signal, this is exactly the same with the conditions of the spatial Fourier transform. And in the process of the formula 8 , it found that the coefficient $\mathrm{K}$ is related to second dispersion, it must meet

$$
\mathrm{K}=\frac{1}{2 \beta_{22} \mathrm{~L}_{2}}
$$

From the above, the time lens system which are composed of input dispersion fiber, output dispersion fiber and phase modulator can achieve the Fourier transform from the input signal in spectrum to the output signal in time domain. And about the principles and conditions for the time lens imaging, Kolner made a detailed discussion ${ }^{[1-2]}$.

\section{Constructing double-lens system to achieve signal shaping and de-noising}

After using a single lens system to achieve the Fourier transform of the time domain, that we can not only do a series of effective treatment to the signal, it is but also need to convert the signal from the time domain to frequency domain. The relationship between Fourier transform and inverse transform are as follows.

$$
\mathrm{F}(f(t))=F(w) \Leftrightarrow F[F(t)]=2 \pi f(-w)
$$

From the above equation, the signal can be converted from the frequency form to the time form by the time lens. Thus we try to build a two-lens system and converse them to the original time-domain signal after the signal is processed.

Signal

Processing
The two-lens system modal is shown as Fig 2.In the current communication system, white noise very commonly exists. However, it is difficult to eliminate white noise which throughout the frequency-domain by conventional signal processing devices, and for ultra short light pulses, especially for those picoseconds femtoseconds optical pulses, the demanding to sensitivity of the device is quite high , but reaction rates of general signal processing device is not so high. However, the narrower the ultra short pulse signals in the time domain, the spectrum will be wider, so that the time-domain waveform signals can be reduced after converting to a wide spectrum of the signal sensitivity of the device, such as using a filter to removal signal of side lobes etc., but in communication system to process the related signal will produce side lobes. Try to use double lens to convert the main lobe of the spectrum to the time domain, which effectively eliminate the ultra short pulse of side lobes.

In the time domain, Gaussian white noise obeys Gaussian distribution, but in the frequency domain, it is uniformly distributed. As a result, in the time domain Gaussian white noise signal has an effect of the disturbances of a signal, but it has little effect on the frequency spectrum of the signal. Assume the Gaussian white noise is $n(t)$, and the original signal is $s(t)$. Gaussian white noise is additive noise. So The input signal which contains Gaussian white noise of $f(t)$ is $\mathrm{n}(\mathrm{t})+\mathrm{s}(\mathrm{t})$, and the Fourier transform is as following :

$$
\mathrm{F}[f(t)]=\int_{-\infty}^{\infty}[s(t)+n(t)] e^{-i w t} d t=F[s(t)]+F[n(t)]
$$

In the above equation, $\mathrm{F}[\mathrm{n}(\mathrm{t})]$ can be effectively treated as a constant, That's to say Gaussian white noise hardly changes the spectrum envelope of the original signal, thus in the time domain we can do the Fourier transformation by the time lens, after that the spectrum of the input signal envelope package will change up to the time domain, and then converted back through doublelens system. When the signal goes through the first lens system, it can be added a band-pass filter to improve noise reduction effect. 
When a delayed signal $s(t)$ produced becomes $s\left(t-t_{0}\right)$, the spectrum is

$$
\mathrm{F}\left(s\left(t-t_{0}\right)\right)=\frac{1}{2 \pi} \int_{-\infty}^{\infty} S(w) e^{i w\left(t-t_{0}\right)} d w=e^{-i w t_{0}} S(w)
$$

Thus, the signal is shifted in time introducing a phase shift in its spectrum, it does not change the shape of the spectral envelope, and so after the two-lens system can be restored $\mathrm{s}(\mathrm{t})$.

Use MATLAB to simulate the following procedure for the double-lens system of Fourier transform functions and Fourier transform functions. On the basis of Fourier transform that a single lens satisfy two dispersion values are equal and the phase shift coefficient with the number, assume the first time provided a lens system, at this time, and dispersion values of the lens system between the second time and the first time is equal, but opposite in sign, so the last signal inversion is not appear. As shown in Figure 3.

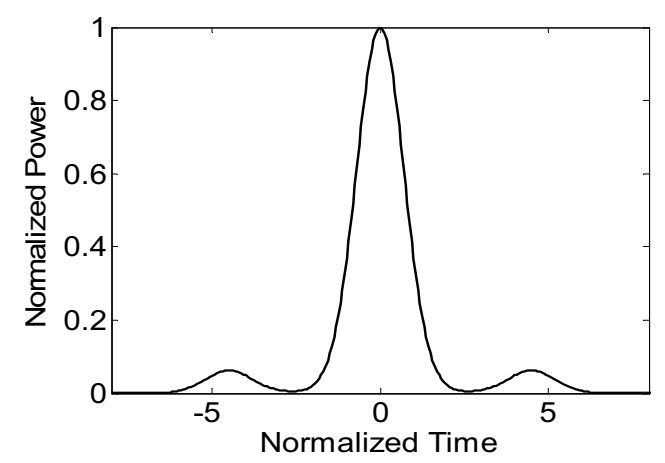

(a) The original signal in time

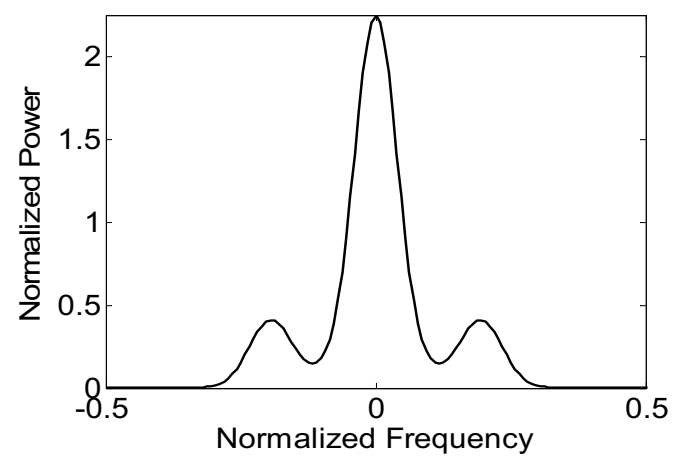

(b) The original signal in spectrum

Figure 3. The waveform and spectrum of the original

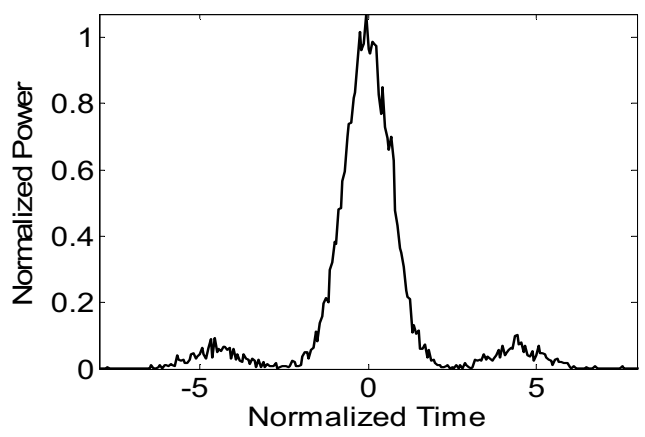

(a) The time signal with side lobe and AWGN

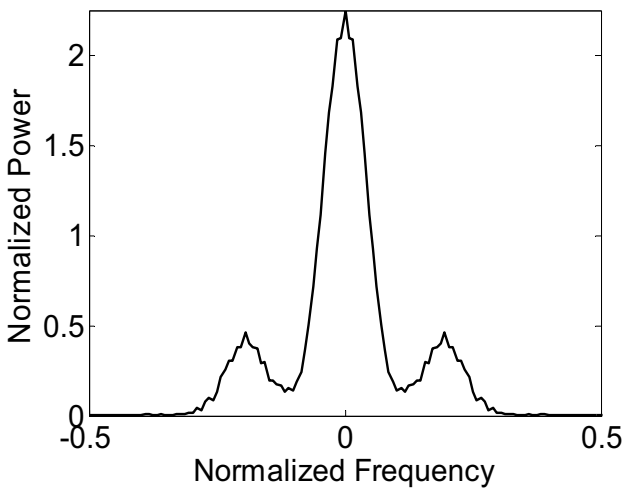

(b) The spectrum of the time signal

Figure 4. The waveform and spectrum of the input signal

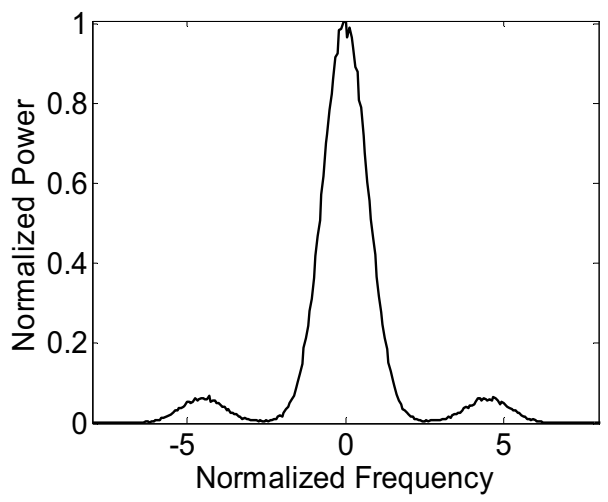

Figure 5. The spectrum of the signal after the first time lens

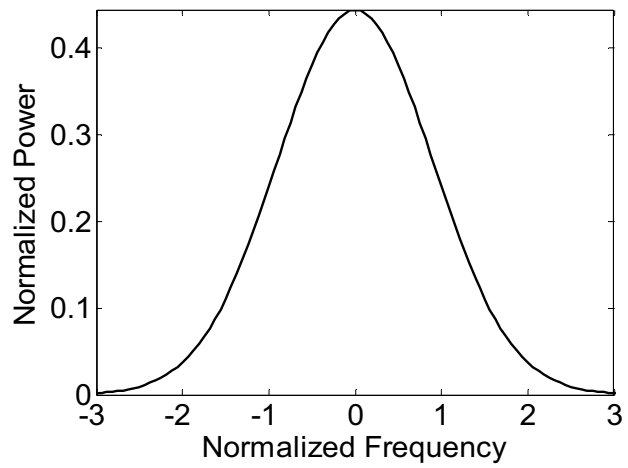

Figure 6. The spectrum after the filter

The Gaussian signal with Gaussian white noise and side lobe as an input signal of the two-lens system, after the first time through the lens into the system, the output waveform and spectrum are shown above, in order to facilitate the observation of comparison, we do the normalization process for variables.

The time-domain waveform of the output is the spectral shape belonging to the original the Fourier transform through the time lens system, which is consistent with the theoretical derivation. At the same time, the spectrum has changed to the corresponding time-domain waveform and scarcely observed original Gaussian white noise. If use an ordinary filter, the original signal side lobe cannot be effectively cut off. However, time-lens will transform the original extremely 
narrow time-domain waveform to a very wide frequency domain, using a common signal filtering can effectively filter in the frequency domain. Setting up a band-pass fiber in the first output of time lens system, the ideal band-pass filter, the transfer function $h(t)=\operatorname{sinc}(a t)$, is used in order to idealized. Finally, the output

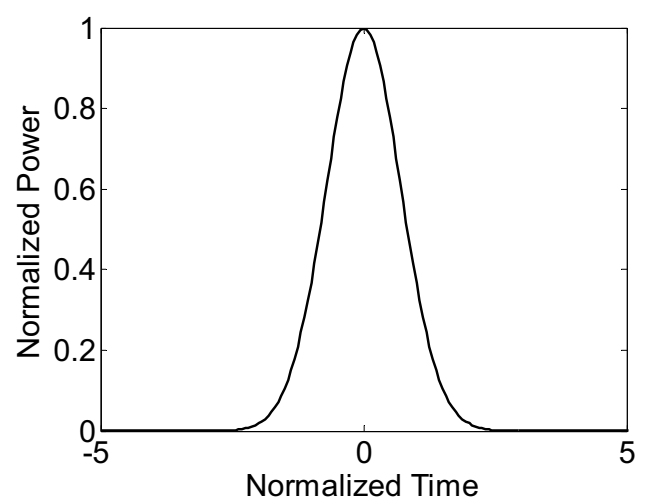

(a) The signal after the processing of double-lens

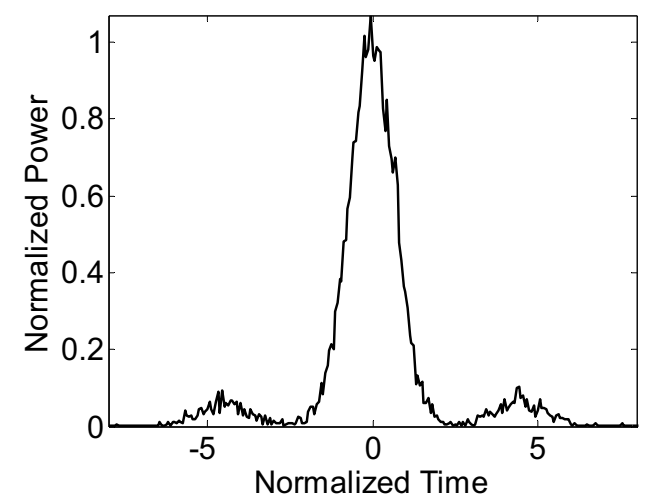

(b) The original signal side lobe and AWGN

Figure 7. The comparison between the original signal and processed signal

waveform and spectrum shown in Figure 7. Its frequency spectrum is Gaussian for a Gaussian pulse. After the noise reduction and side lobe amputated, the second time lens system is used to recovery ideal signal pulses to be obtained. The final output signal waveform shown in Figure 9, and compared with the original signal noise and side lobe. The above theoretical description and simulation visually demonstrate that the treatment effect of signal pulse, the effective realization of the noise filter, used the double-lens system by Fourier transform [10].

\section{Conclusions}

In this paper, we use the Fourier transform lens function of time to achieve a narrow pulse de-noising and cut side lobe. It is effective to improve the white noise witch exist in communication system commonly, and side lobe effect when we process the signal. But because it is the simulation to the signal, the experimental results will be affected by signal time window and sampling points, it can be not accurately show the relationship between narrow band time domain and frequency domain, and using the ideal band pass filter, so the actual effect of the theory can not be reached the theoretical results.

\section{References}

1. B.H. Kolner, M. Nazarathy, Opt. Lett., 14, 630632(1989)

2. B. H. Kolner, IEEE J. Quantum Electron., 30, 1951 1963(1994)

3. A.A. Godil, Auld B A, Bloom D M, Appl. Phys. Lett, 62, 1047-1479(1993)

4. R. Salem, M.A. Foster, A.C. Turner, D.F. Geraghty, M.s Lipson and A. L. Gaeta, Opt. Lett., 33, 1047- 1049(2008)

5. C.V. Bennett, B.H. Kolner, IEEE J. Quantum Electron., 36, 430-437(2000)

6. T.Ng T., F. Parmigiani, M. Ibsen, Zhang ZH W, IEEE Photon. Technol. Lett., 20, 1097-1099(2008)

7. J. A. Howe, C. Xu , Digest of the LEOS Summer Topical Meetings( 2005)

8. M. T. Kauffman, W. C. Banyai, A.A. Godil and D. M Bloom, Appl. Phys. Lett., 64, 270-272(1994)

9. A. A. Godil, B. A. Auld and D. M.Bloom., IEEE J. Quantum Electron., 30, 827-837(1994)

10. S. Kumar, Opt. Lett, 32, 346-348(2007) 